\title{
SEARCH FOR GRAVITATIONAL-WAVE INSPIRAL SIGNALS ASSOCIATED WITH SHORT GAMMA-RAY BURSTS DURING LIGO'S FIFTH AND VIRGO'S FIRST SCIENCE RUN
}

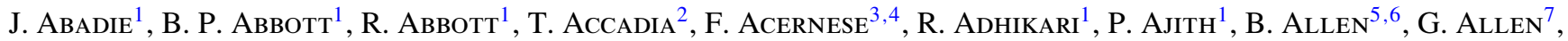
E. Amador Ceron ${ }^{6}$, R. S. Amin ${ }^{8}$, S. B. Anderson ${ }^{1}$, W. G. Anderson ${ }^{6}$, F. Antonucci ${ }^{9}$, S. Aoudia ${ }^{10}$, M. A. Arain ${ }^{11}$,

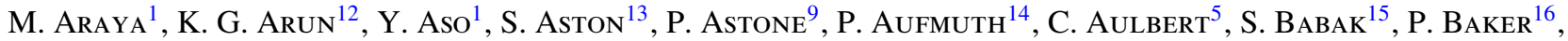

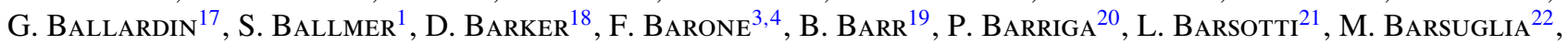

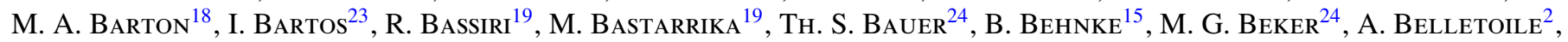
M. Benacquista ${ }^{25}$, J. Betzwieser ${ }^{1}$, P. T. Beyersdorf ${ }^{26}$, S. Bigotta ${ }^{27,28}$, I. A. Bilenko ${ }^{29}$, G. Billingsley ${ }^{1}$, S. Birindelli ${ }^{10}$, R. Biswas ${ }^{6}$, M. A. Bizouard ${ }^{12}$, E. Black ${ }^{1}$, J. K. Blackburn ${ }^{1}$, L. Blackburn ${ }^{21}$, D. Blair ${ }^{20}$, B. Bland ${ }^{18}$, M. Blom ${ }^{24}$,

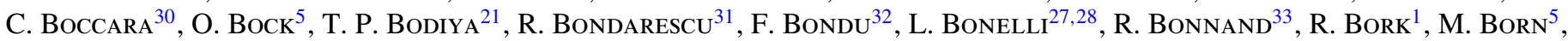

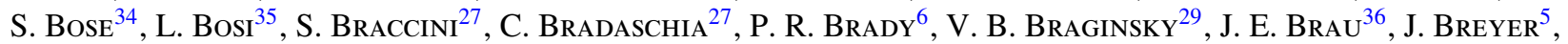
D. O. Bridges $^{37}$, A. BrilletT ${ }^{10}$, M. BrinkmanN ${ }^{5}$, V. Brisson ${ }^{12}$, M. BritzGer ${ }^{5}$, A. F. Brooks ${ }^{1}$, D. A. Brown ${ }^{38}$, R. BudZYŃski ${ }^{39}$, T. Bulik ${ }^{40,41}$, A. Bullington ${ }^{7}$, H. J. Bulten ${ }^{24,42}$, A. Buonanno ${ }^{43}$, J. Burguet-Castell ${ }^{6}$, O. Burmeister ${ }^{5}$, D. Buskulic ${ }^{2}$, C. Buy ${ }^{22}$, R. L. Byer ${ }^{7}$, L. Cadonati ${ }^{44}$, G. CaGnOli ${ }^{45}$, J. Cain ${ }^{46}$, E. Calloni ${ }^{3,47}$, J. B. Camp ${ }^{48}$, E. Campagna ${ }^{45,49}$,

J. Cannizzo ${ }^{48}$, K. C. Cannon ${ }^{1}$, B. Canuel ${ }^{17}$, J. CaO ${ }^{21}$, C. D. Capano ${ }^{38}$, F. Carbognani ${ }^{17}$, L. Cardenas ${ }^{1}$, S. Caudill ${ }^{8}$, M. Cavaglià ${ }^{46}$, F. Cavalier ${ }^{12}$, R. Cavalieri ${ }^{17}$, G. Cella $^{27}$, C. Cepeda ${ }^{1}$, E. Cesarini ${ }^{49}$, T. Chalermsongsak ${ }^{1}$, E. Chalkley $^{19}$, P. Charlton ${ }^{50}$, E. Chassande-Mottin ${ }^{22}$, S. ChatteriI ${ }^{1}$, S. ChelKowski ${ }^{13}$, Y. Chen $^{51}$, A. ChinCarini ${ }^{52}$, N. Christensen ${ }^{53}$,

S. S. Y. Chua ${ }^{54}$, C. T. Y. Chung ${ }^{55}$, D. Clark $^{7}$, J. Clark ${ }^{56}$, J. H. Clayton ${ }^{6}$, F. ClevaA ${ }^{10}$, E. Coccia ${ }^{57,58}$, C. N. Colacino ${ }^{27}$, J. Colas ${ }^{17}$, A. Colla ${ }^{9,59}$, M. Colombini ${ }^{59}$, R. Conte $^{4}$, D. Cook ${ }^{18}$, T. R. C. Corbitt $^{21}$, N. Cornish ${ }^{16}$, A. Corsi ${ }^{9}$, J.-P. Coulon ${ }^{10}$,

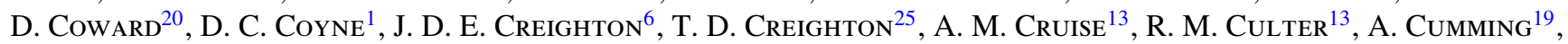

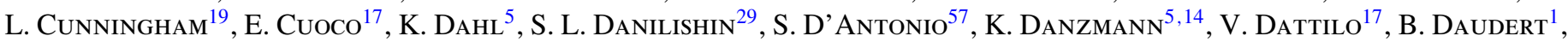
M. Davier ${ }^{12}$, G. Davies ${ }^{56}$, E. J. DaW ${ }^{60}$, R. DaY ${ }^{17}$, T. DaYAnga ${ }^{34}$, R. De Rosa ${ }^{3,47}$, D. DeBra ${ }^{7}$, J. Degallaix ${ }^{5}$, M. Del Prete ${ }^{27,61}$, V. Dergachev ${ }^{62}$, R. DeSalvo ${ }^{1}$, S. Dhurandhar ${ }^{63}$, L. Di Fiore ${ }^{3}$, A. Di Lieto ${ }^{27,28}$, M. Di Paolo Emilio ${ }^{57,64}$, A. Di Virgilio ${ }^{27}$,

M. Díaz ${ }^{25}$, A. Dietz ${ }^{2}$, F. Donovan ${ }^{21}$, K. L. Dooley ${ }^{11}$, E. E. Doomes ${ }^{65}$, M. Drago ${ }^{66,67}$, R. W. P. Drever ${ }^{68}$, J. Driggers ${ }^{1}$,

J. Dueck ${ }^{5}$, I. Duke ${ }^{21}$, J.-C. Dumas ${ }^{20}$, M. Edgar ${ }^{19}$, M. Edwards ${ }^{56}$, A. Effler ${ }^{18}$, P. Ehrens ${ }^{1}$, T. Etzel ${ }^{1}$, M. Evans ${ }^{21}$, T. Evans $^{37}$, V. FAFOnE ${ }^{57,58}$, S. Fairhurst ${ }^{56}$, Y. Faltas ${ }^{11}$, Y. FAN ${ }^{20}$, D. FAZI ${ }^{1}$, H. FehrmanN ${ }^{5}$, I. Ferrante ${ }^{27,28}$, F. FideCARo ${ }^{27,28}$,

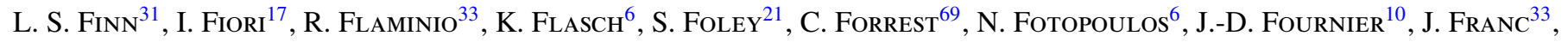
S. Frasca ${ }^{9,59}$, F. Frascon ${ }^{27}$, M. Frede ${ }^{5}$, M. Frei ${ }^{70}$, Z. Frei ${ }^{71}$, A. Freise ${ }^{13}$, R. Frey $^{36}$, T. T. Fricke ${ }^{8}$, D. Friedrich ${ }^{5}$, P. Fritschel ${ }^{21}$, V. V. Frolov ${ }^{37}$, P. Fulda ${ }^{13}$, M. FyfFe ${ }^{37}$, M. Galimberti ${ }^{33}$, L. Gammaitoni ${ }^{35,72}$, J. A. Garofoli ${ }^{38}$, F. Garufi ${ }^{3,47}$, G. Gemme ${ }^{52}$, E. Genin ${ }^{17}$, A. Gennai ${ }^{27}$, S. Ghosh ${ }^{34}$, J. A. Giaime ${ }^{8,37}$, S. Giampanis ${ }^{5}$, K. D. Giardina ${ }^{37}$, A. Giazotto ${ }^{27}$, E. Goetz ${ }^{62}$, L. M. Goggin ${ }^{6}$, G. GonzÁleZ $^{8}$, S. Goßler $^{5}$, R. GouatY $^{2}$, M. Granata $^{22}$, A. Grant ${ }^{19}$, S. Gras $^{20}$, C. GraY ${ }^{18}$, R. J. S. Greenhalgh ${ }^{73}$, A. M. Gretarsson ${ }^{74}$, C. Greverie $^{10}$, R. Grosso ${ }^{25}$, H. Grote ${ }^{5}$, S. Grunewald ${ }^{15}$,

G. M. Guidi ${ }^{45,49}$, E. K. Gustafson ${ }^{1}$, R. Gustafson ${ }^{62}$, B. Hage ${ }^{14}$, J. M. Hallam ${ }^{13}$, D. Hammer ${ }^{6}$, G. D. Hammond ${ }^{19}$, C. HanNa ${ }^{1}$,

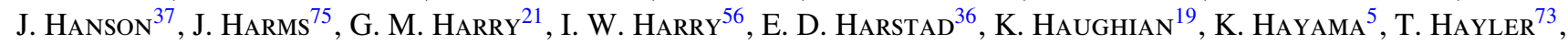

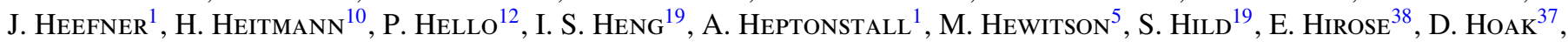

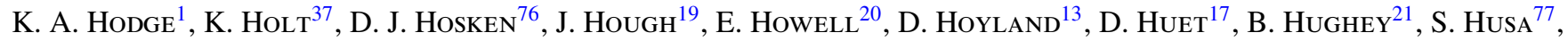

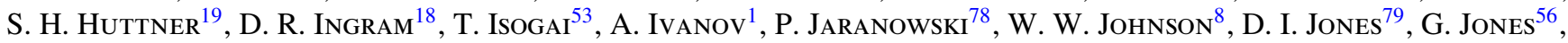
R. Jones ${ }^{19}$, L. Ju ${ }^{20}$, P. Kalmus ${ }^{1}$, V. Kalogera ${ }^{80}$, S. Kandhasamy ${ }^{75}$, J. Kanner ${ }^{43}$, E. Katsavounidis ${ }^{21}$, K. Kawabe ${ }^{18}$, S. Kawamura ${ }^{81}$, F. Kawazoe ${ }^{5}$, W. Kells ${ }^{1}$, D. G. Keppel ${ }^{1}$, A. Khalaidoviski ${ }^{5}$, F. Y. Khalili ${ }^{29}$, R. Khan ${ }^{23}$, E. Khazanov ${ }^{82}$,

H. Kim ${ }^{5}$, P. J. King ${ }^{1}$, J. S. KisseL ${ }^{8}$, S. KlimenKo ${ }^{11}$, K. KoKeyama ${ }^{81}$, V. Kondrashov ${ }^{1}$, R. KopparaPU ${ }^{31}$, S. Koranda ${ }^{6}$,

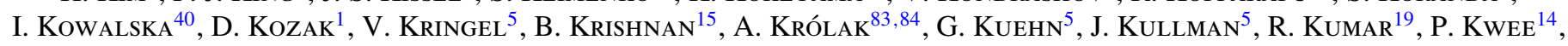

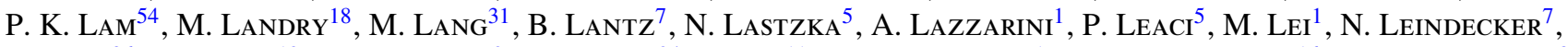

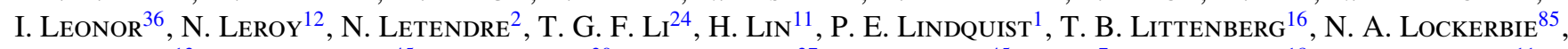

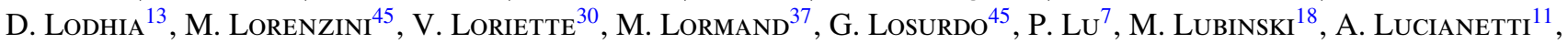

H. LÜCK ${ }^{5,14}$, A. Lundgren ${ }^{38}$, B. MachenschalK ${ }^{5}$, M. MacInnis ${ }^{21}$, M. Mageswaran ${ }^{1}$, K. Mailand ${ }^{1}$, E. Majorana ${ }^{9}$, C. MaK $^{1}$,

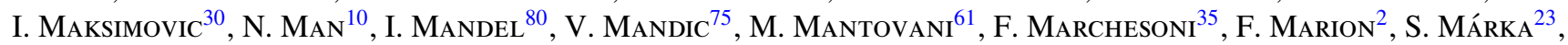

Z. Márka ${ }^{23}$, A. Markosyan ${ }^{7}$, J. Markowitz ${ }^{21}$, E. Maros ${ }^{1}$, J. Marque ${ }^{17}$, F. Martelli ${ }^{45}, 49$, I. W. Martin ${ }^{19}$, R. M. Martin ${ }^{11}$, J. N. Marx ${ }^{1}$, K. Mason ${ }^{21}$, A. Masserot ${ }^{2}$, F. Matichard ${ }^{8,21}$, L. Matone ${ }^{23}$, R. A. Matzner ${ }^{70}$, N. Mavalvala ${ }^{21}$,

R. McCarthy ${ }^{18}$, D. E. McClelland ${ }^{54}$, S. C. McGuire ${ }^{65}$, G. McIntyre ${ }^{1}$, D. J. A. McKechan ${ }^{56}$, M. Mehmet ${ }^{5}$, A. Melatos $^{55}$, A. C. Melissinos ${ }^{69}$, G. Mendell ${ }^{18}$, D. F. Menéndez ${ }^{31}$, R. A. Mercer ${ }^{6}$, L. Merill ${ }^{20}$, S. Meshioov ${ }^{1}$, C. Messenger ${ }^{5}$, M. S. Meyer ${ }^{37}$, H. Miao ${ }^{20}$, C. Michel ${ }^{33}$, L. Milano ${ }^{3,47}$, J. Milleer ${ }^{19}$, Y. Minenkov ${ }^{56}$, Y. Mino $^{51}$, S. Mitra ${ }^{1}$,

V. P. Mitrofanov ${ }^{29}$, G. Mitselmakher ${ }^{11}$, R. Mittleman ${ }^{21}$, O. Miyakawa ${ }^{1}$, B. MoE ${ }^{6}$, M. Mohan ${ }^{17}$, S. D. Mohanty ${ }^{25}$, S. R. P. Mohapatra ${ }^{44}$, J. Moread ${ }^{30}$, G. Moreno ${ }^{18}$, N. Morgado ${ }^{33}$, A. Morgia ${ }^{57,58}$, K. Mors ${ }^{5}$, S. Mosca ${ }^{3,47}$, V. Moscatelli ${ }^{9}$,

K. Mossavi ${ }^{5}$, B. Mours $^{2}$, C. Mowlowry ${ }^{54}$, G. Mueller ${ }^{11}$, S. Mukherjee ${ }^{25}$, A. Mullavey ${ }^{54}$, H. Müller-Ebhardt ${ }^{5}$,

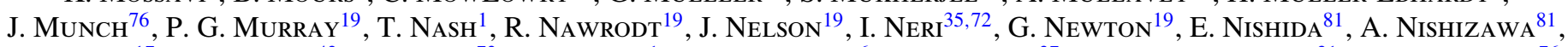
F. Nocera ${ }^{17}$, E. Ochsner ${ }^{43}$, J. O'Dell ${ }^{73}$, G. H. Ogin ${ }^{1}$, R. Oldenburg ${ }^{6}$, B. O’Reilly ${ }^{37}$, R. O’Shaughnessy ${ }^{31}$, D. J. Ottaway ${ }^{76}$, 
R. S. Ottens ${ }^{11}$, H. Overmier ${ }^{37}$, B. J. Owen ${ }^{31}$, A. Page ${ }^{13}$, G. Pagliaroli ${ }^{57,58}$, C. Palomba ${ }^{9}$, Y. Pan ${ }^{43}$, C. Pankow ${ }^{11}$,

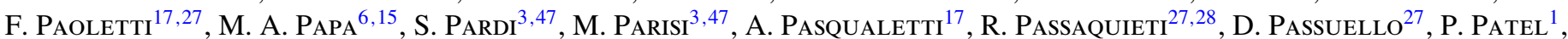
D. Pathak ${ }^{56}$, M. Pedraza ${ }^{1}$, L. PeKowsk $^{38}$, S. PenN $^{86}$, C. Peralta $^{15}$, A. Perreca $^{13}$, G. Persichetti $^{3,47}$, M. Pichot $^{10}$,

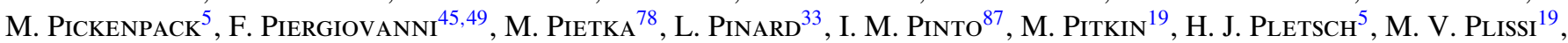
R. Poggiani ${ }^{27,28}$, F. Postiglione ${ }^{4}$, M. Prato ${ }^{52}$, V. Predoi ${ }^{56}$, M. Principe ${ }^{87}$, R. Prix ${ }^{5}$, G. A. Prodi ${ }^{88,89}$, L. Prokhorov ${ }^{29}$,

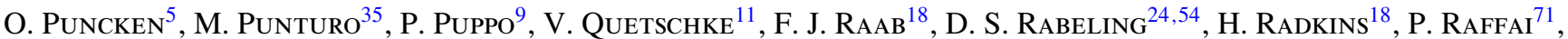

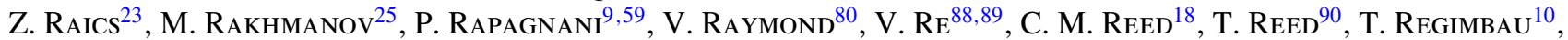
H. Rehbein ${ }^{5}$, S. Reid ${ }^{19}$, D. H. ReItze ${ }^{11}$, F. Ricci ${ }^{9,59}$, R. Riesen ${ }^{37}$, K. Riles ${ }^{62}$, P. Roberts ${ }^{91}$, N. A. Robertson ${ }^{1,19}$, F. Robinet ${ }^{12}$, C. Robinson ${ }^{56}$, E. L. Robinson ${ }^{15}$, A. RocchI ${ }^{57}$, S. RodDY ${ }^{37}$, C. Röver ${ }^{5}$, L. Rolland ${ }^{2}$, J. Rollins ${ }^{23}$, J. D. Romano ${ }^{25}$,

R. Romano ${ }^{3,4}$, J. H. Romie ${ }^{37}$, D. Rosińska ${ }^{92}$, S. Rowan ${ }^{19}$, A. RÜDIGER ${ }^{5}$, P. RUgGi ${ }^{17}$, K. Ryan ${ }^{18}$, S. SAKata ${ }^{81}$, F. SALEMI ${ }^{5}$, L. Sammut ${ }^{55}$, L. Sancho de la Jordana ${ }^{77}$, V. Sandberg ${ }^{18}$, V. Sannibale ${ }^{1}$, L. Santamaría ${ }^{15}$, G. Santostasi ${ }^{93}$, S. Saraf ${ }^{94}$, P. Sarin ${ }^{21}$, B. Sassolas ${ }^{33}$, B. S. SathyapraKash ${ }^{56}$, S. Sato ${ }^{81}$, M. SatTerthWaite ${ }^{54}$, P. R. SAulson ${ }^{38}$, R. SAVAGE ${ }^{18}$, R. Schilling ${ }^{5}$, R. Schnabel ${ }^{5}$, R. Schofield ${ }^{36}$, B. SchulZ ${ }^{5}$, B. F. Schutz ${ }^{15,56}$, P. Schwinberg ${ }^{18}$, J. Scott ${ }^{19}$, S. M. ScotT ${ }^{54}$, A. C. Searle ${ }^{1}$, F. Seifert ${ }^{1,5}$, D. Sellers ${ }^{37}$, A. S. Sengupta ${ }^{1}$, D. Sentenac ${ }^{17}$, A. Sergeev ${ }^{82}$, B. Shapiro ${ }^{21}$, P. Shawhan ${ }^{43}$, D. H. Shoemaker ${ }^{21}$, A. Sibley ${ }^{37}$, X. Siemens ${ }^{6}$, D. SigG ${ }^{18}$, A. M. Sintes ${ }^{77}$, G. Skelton ${ }^{6}$, B. J. J. Slagmolen ${ }^{54}$, J. SLutsky ${ }^{8}$, J. R. Smith ${ }^{38}$, M. R. Smith ${ }^{1}$, N. D. Smith ${ }^{21}$, K. SomiYa ${ }^{51}$, B. Sorazu ${ }^{19}$, A. J. STeIN ${ }^{21}$, L. C. STEIN ${ }^{21}$, S. StePlewski ${ }^{34}$, A. Stochino ${ }^{1}$, R. Stone ${ }^{25}$, K. A. Strain ${ }^{19}$, S. Strigin ${ }^{29}$, A. Stroeer ${ }^{48}$, R. Sturani ${ }^{45,49}$, A. L. Stuver ${ }^{37}$,

T. Z. Summerscales ${ }^{91}$, M. Sung ${ }^{8}$, S. Susmithan ${ }^{20}$, P. J. Sutton ${ }^{56}$, B. Swinkels ${ }^{17}$, G. P. Szokoly ${ }^{71}$, D. TalukdeR ${ }^{34}$,

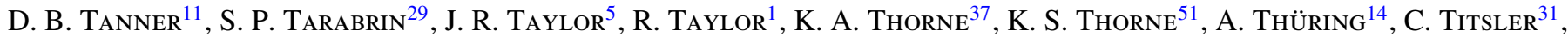
K. V. Tokmakov ${ }^{19,85}$, A. Toncelli ${ }^{27,28}$, M. Tonelli ${ }^{27,28}$, C. Torres $^{37}$, C. I. Torrie ${ }^{1,19}$, E. Tournefier ${ }^{2}$, F. Travasso $^{35,72}$, G. Traylor ${ }^{37}$, M. Trias ${ }^{77}$, J. Trummer ${ }^{2}$, L. Turner ${ }^{1}$, D. Ugolini ${ }^{95},{\text { K. } \text { UrbaneK }^{7}, \text { H. Vahlbruch }^{14} \text {, G. Vajente }}^{27,28}$, M. Vallisneri ${ }^{51}$, J. F. J. van den Brand ${ }^{24,42}$, C. Van Den Broeck ${ }^{56}$, S. van der Putten ${ }^{24}$, M. V. van der Sluys ${ }^{80}$, S. Vass $^{1}$,

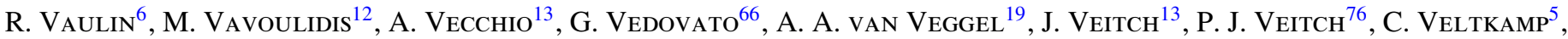
D. Verkindt ${ }^{2}$, F. Vetrano ${ }^{45,49}$, A. Viceré ${ }^{45,49}$, A. Villar ${ }^{1}$, J.-Y. Vinet ${ }^{10}$, H. Vocca ${ }^{35}$, C. Vorvick ${ }^{18}$, S. P. Vyachanin ${ }^{29}$, S. J. Waldman ${ }^{21}$, L. WAllace ${ }^{1}$, A. WANner ${ }^{5}$, R. L. WARD ${ }^{1}$, M. Was ${ }^{12}$, P. Wei $^{38}$, M. WeinerT ${ }^{5}$, A. J. Weinstein ${ }^{1}$, R. Weiss ${ }^{21}$, L. Wen ${ }^{20,51}$, S. Wen $^{8}$, P. Wessels ${ }^{5}$, M. West ${ }^{38}$, T. WestPhal ${ }^{5}$, K. Wette ${ }^{54}$, J. T. Whelan ${ }^{96}$, S. E. Whitcomb ${ }^{1}$,

B. F. Whiting ${ }^{11}$, C. Wilkinson ${ }^{18}$, P. A. Willems ${ }^{1}$, H. R. Williams ${ }^{31}$, L. Williams ${ }^{11}$, B. Willke ${ }^{5,14}$, I. Wilmut ${ }^{73}$,

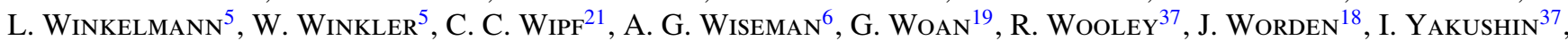

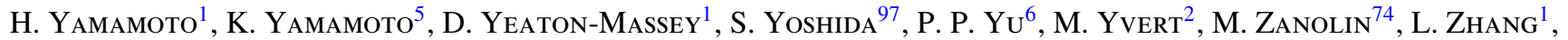
Z. ZHANG ${ }^{20}$, C. ZHAO ${ }^{20}$, N. Zotov ${ }^{90}$, M. E. ZuCKeR ${ }^{21}$, AND J. ZWEIZIG ${ }^{1}$

(The LiGo Scientific Collaboration \& The Virgo Collaboration)

${ }^{1}$ LIGO-California Institute of Technology, Pasadena, CA 91125, USA

${ }^{2}$ Laboratoire d'Annecy-le-Vieux de Physique des Particules (LAPP), Université de Savoie, CNRS/IN2P3, F-74941 Annecy-le-Vieux, France

${ }^{3}$ INFN, Sezione di Napoli, Complesso Universitario di Monte Sant'Angelo, I-80126 Napoli, Italy

${ }^{4}$ Università di Salerno, I-84084 Fisciano (Salerno), Italy

${ }^{5}$ Albert-Einstein-Institut, Max-Planck-Institut für Gravitationsphysik, D-30167 Hannover, Germany

${ }^{6}$ University of Wisconsin-Milwaukee, Milwaukee, WI 53201, USA

${ }^{7}$ Stanford University, Stanford, CA 94305, USA

${ }^{8}$ Louisiana State University, Baton Rouge, LA 70803, USA

${ }^{9}$ INFN, Sezione di Roma, I-00185 Roma, Italy

${ }^{10}$ Université Nice-Sophia-Antipolis, CNRS, Observatoire de la Côte d'Azur, F-06304 Nice, France

${ }^{11}$ University of Florida, Gainesville, FL 32611, USA

${ }^{12}$ LAL, Université Paris-Sud, IN2P3/CNRS, F-91898 Orsay, France

${ }^{13}$ University of Birmingham, Birmingham, B15 2TT, UK

${ }^{14}$ Leibniz Universität Hannover, D-30167 Hannover, Germany

${ }^{15}$ Albert-Einstein-Institut, Max-Planck-Institut für Gravitationsphysik, D-14476 Golm, Germany

${ }^{16}$ Montana State University, Bozeman, MT 59717, USA

${ }^{17}$ European Gravitational Observatory (EGO), I-56021 Cascina (Pi), Italy

${ }^{18}$ LIGO-Hanford Observatory, Richland, WA 99352, USA

${ }^{19}$ University of Glasgow, Glasgow, G12 8QQ, UK

${ }^{20}$ University of Western Australia, Crawley, WA 6009, Australia

${ }^{21}$ LIGO-Massachusetts Institute of Technology, Cambridge, MA 02139, USA

${ }^{22}$ AstroParticule et Cosmologie (APC), CNRS: UMR7164-IN2P3-Observatoire de Paris-Université Denis Diderot-Paris 7-CEA : DSM/IRFU, France ${ }^{23}$ Columbia University, New York, NY 10027, USA

${ }^{24}$ Nikhef, National Institute for Subatomic Physics, P. O. Box 41882, 1009 DB, Amsterdam, The Netherlands

${ }^{25}$ The University of Texas at Brownsville and Texas Southmost College, Brownsville, TX 78520, USA

${ }^{26}$ San Jose State University, San Jose, CA 95192, USA

${ }^{27}$ INFN, Sezione di Pisa, I-56127 Pisa, Italy

${ }^{28}$ Università di Pisa, I-56127 Pisa; Italy

${ }^{29}$ Moscow State University, Moscow, 119992, Russia

${ }^{30}$ ESPCI, CNRS, F-75005 Paris, France

31 The Pennsylvania State University, University Park, PA 16802, USA

${ }^{32}$ Institut de Physique de Rennes, CNRS, Université de Rennes 1, 35042 Rennes, France

${ }^{33}$ Laboratoire des Matériaux Avancés (LMA), IN2P3/CNRS, F-69622 Villeurbanne, Lyon, France

${ }^{34}$ Washington State University, Pullman, WA 99164, USA

35 INFN, Sezione di Perugia, I-6123 Perugia, Italy

${ }^{36}$ University of Oregon, Eugene, OR 97403, USA 


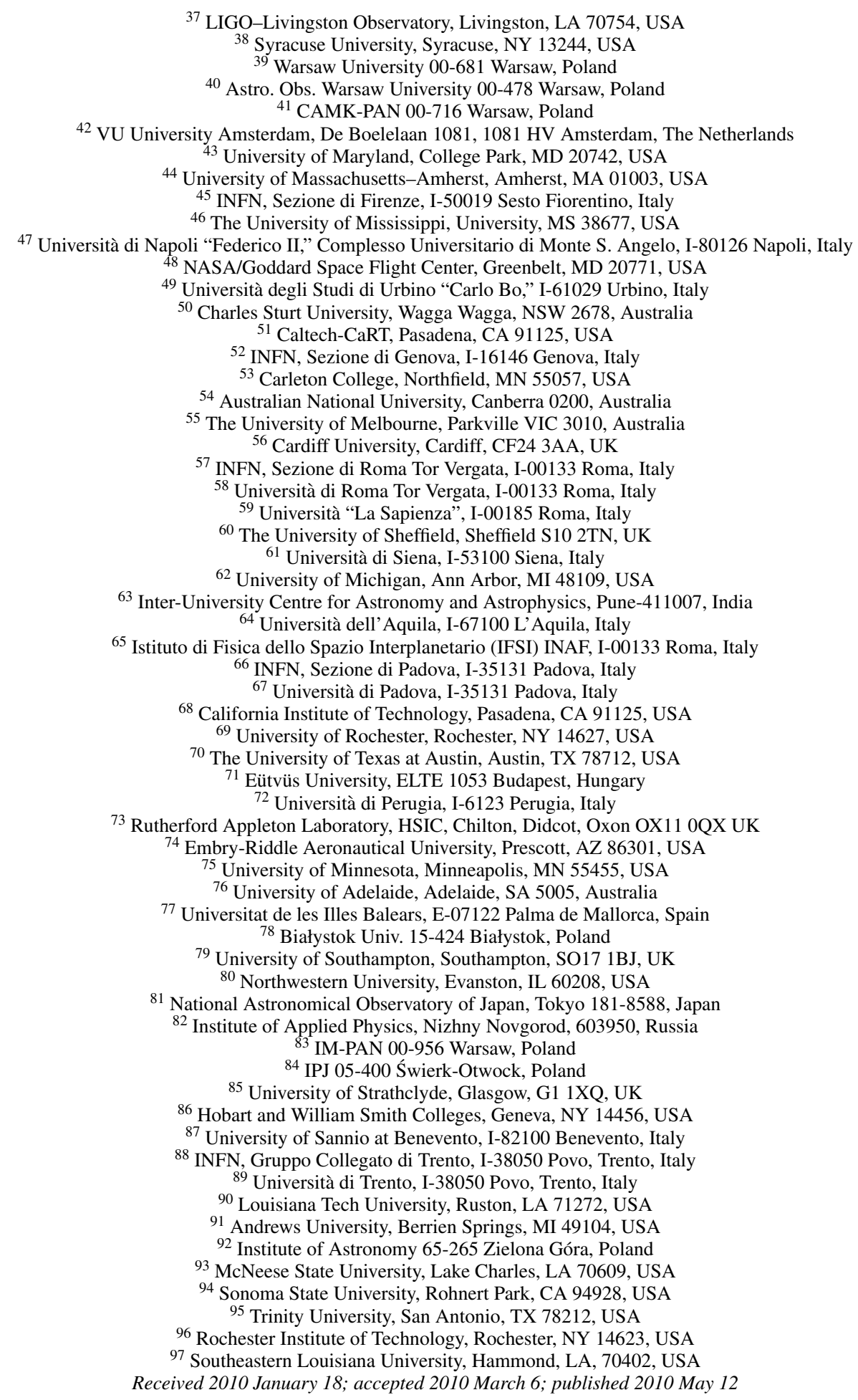

\section{ABSTRACT}

Progenitor scenarios for short gamma-ray bursts (short GRBs) include coalescenses of two neutron stars or a neutron star and black hole, which would necessarily be accompanied by the emission of strong gravitational waves. We present a search for these known gravitational-wave signatures in temporal and directional coincidence with 22 GRBs that had sufficient gravitational-wave data available in multiple instruments during LIGO's fifth science run, S5, and Virgo's first science run, VSR1. We find no statistically significant gravitational-wave candidates within a $[-5,+1) \mathrm{s}$ window around the trigger time of any GRB. Using the Wilcoxon-Mann-Whitney $U$-test, we find no evidence for an excess of weak gravitational-wave signals in our sample of GRBs. We exclude neutron star-black hole progenitors to a median $90 \%$ confidence exclusion distance of $6.7 \mathrm{Mpc}$.

Key words: binaries: close - gamma-ray burst: general - gravitational waves 


\section{INTRODUCTION}

The past decade has seen dramatic progress in the understanding of gamma-ray bursts (GRBs), intense flashes of $\gamma$-rays that are observed to be isotropically distributed over the sky (see, e.g., Klebesadel et al. 1973; Mészáros 2006, and references therein). The short-time variability of the bursts indicates that the sources are very compact. They are observed directly by $\gamma$-ray and X-ray satellites in the Interplanetary Network such as HETE, Swift, Konus-Wind, INTEGRAL, and Fermi (see Ricker et al. 2003; Gehrels et al. 2004; Aptekar et al. 1995; Winkler et al. 2003; Atwood et al. 2009, and references therein).

GRBs are usually divided into two types (see Kouveliotou et al. 1993; Gehrels et al. 2006), distinguished primarily by the duration of the prompt burst. Long-duration bursts, with a duration of $\gtrsim 2 \mathrm{~s}$, are generally associated with hypernova explosions in star-forming galaxies. Several nearby long GRBs have been spatially and temporally coincident with core-collapse supernovae as observed in the optical (Campana et al. 2006; Galama et al. 1998; Hjorth et al. 2003; Malesani et al. 2004). Followup observations by X-ray, optical, and radio telescopes of the sky near GRBs have yielded detailed measurements of afterglows from more than 500 GRBs to date; some of these observations resulted in strong host galaxy candidates, which allowed redshift determination for more than 200 bursts (Greiner 2009).

Short GRBs, with a duration $\lesssim 2 \mathrm{~s}$, are thought to originate primarily in the coalescence of a neutron star (NS) with another compact object (see, e.g., Nakar 2007, and references therein), such as an NS or black hole (BH). There is growing evidence that finer distinctions may be drawn between bursts (Zhang et al. 2007; Bloom et al. 2008); for example, it is estimated that up to $\sim 15 \%$ of short GRBs could be associated with soft gamma repeaters (Nakar et al. 2006; Chapman et al. 2009), which emit bursts of X-rays and gamma rays at irregular intervals with lower fluence than compact binary coalescence engines (Hurley et al. 2005; Palmer et al. 2005).

In the compact binary coalescence model of short GRBs, an NS and compact companion in otherwise stable orbit lose energy to gravitational waves and inspiral. The neutron star(s) tidally disrupt shortly before coalescence, providing matter, some of which is ejected in relativistic jets. The prompt $\gamma$-ray emission is widely thought to be created by internal shocks, the interaction of outgoing matter shells at different velocities, while the afterglow is thought to be created by external shocks, the interaction of the outflowing matter with the interstellar medium (Mészáros 2006; Nakar 2007). If the speed of gravitational radiation equals the speed of light as we expect, then for an observer in the cone of the collimated outflow, the gravitational-wave inspiral signal will arrive a few seconds before the electromagnetic signal from internal shocks. Several semianalytical calculations of the final stages of an NS-BH inspiral show that the majority of matter plunges onto the $\mathrm{BH}$ within $1 \mathrm{~s}$ (Davies et al. 2005). Numerical simulations on the mass transfer suggest a timescale of milliseconds (Shibata \& Taniguchi 2008) or some seconds at maximum (Faber et al. 2006). Also, it has been found in simulations that the vast majority of the NS matter is accreted onto the BH directly and promptly (within hundreds of milliseconds) without a torus that gets accreted later (Rosswog 2007; Etienne et al. 2008).

Compact binary coalescence is anticipated to generate strong gravitational waves in the sensitive frequency band of
Earth-based gravitational-wave detectors (Thorne 1987). The direct detection of gravitational waves associated with a short GRB would provide direct evidence that the progenitor is indeed a compact binary; with such a detection it would be possible to measure component masses (Cutler \& Flanagan 1994; Finn \& Chernoff 1993), measure component spins (Poisson \& Will 1995), constrain NS equations of state (Flanagan \& Hinderer 2008; Read et al. 2009), test general relativity in the strong-field regime (Will 2005), and measure calibration-free luminosity distance (Nissanke et al. 2009), which is a measurement of the Hubble expansion and dark energy.

In this paper, we report on a search for gravitational-wave inspiral signals associated with the short GRBs that occurred during the fifth science run (S5) of LIGO (Abbott et al. 2009a), from 2005 November 4 to 2007 September 30, and the first science run (VSR1) of Virgo (Acernese et al. 2008), from 2007 May 18 to 2007 September 30. S5 represents the combined operation of the three LIGO detectors, one Michelson interferometer with $4 \mathrm{~km}$ long orthogonal arms at Livingston, LA, USA, named L1, and two interferometers located at Hanford, WA, USA, named $\mathrm{H} 1$ and $\mathrm{H} 2$, with lengths of $4 \mathrm{~km}$ and $2 \mathrm{~km}$, respectively. VSR1 represents the operation of the Virgo interferometer located at Cascina, Italy, named V1, which has a length of $3 \mathrm{~km}$. During the S5/VSR1 joint run, 212 GRBs were discovered by different satellite missions (39 of them during VSR1 times), 33 of which we classified as search targets (8 of them in VSR1 times). See Section 2.2 for more details on the selected GRBs.

A similar search in the same LIGO/Virgo data set was performed in Abbott et al. (2010), looking for short-duration gravitational-wave bursts in association with 137 GRBs recorded during S5/VSR1, both long and short. The analysis reported upper limits on the strain of a generic burst of circularly polarized gravitational radiation, predominantly at the detectors' most sensitive frequencies. These were translated into lower limits in distance by assuming that $0.01 M_{\odot}$ is converted into isotropically emitted gravitational waves. In contrast, the search described in this paper does not make any assumption on the polarization of the gravitational waves and searches for the specific signals expected from binary coalescenses. Importantly, the present search can distinguish a coalescence signal from other models and estimate the progenitor parameters.

The remainder of the paper is organized as follows. In Section 2, we discuss the set of GRBs we chose for this analysis and outline our analysis methods. In Section 3, we present the results and astrophysical implications for the GRBs in our sample.

\section{SEARCH METHODS}

\subsection{Experimental Setup}

The binary coalescence model suggests that the time delay between the arrival of a gravitational wave and the arrival of the subsequent electromagnetic burst, referred to as trigger time, is a few seconds. We assessed uncertainties in reported trigger times and quantization in our own analysis along integer second boundaries, finding that these each contribute less than $1 \mathrm{~s}$. For example, when the Swift BAT instrument determines that the count rate has risen above a threshold, it waits for the maximum to pass, checking with a $320 \mathrm{~ms}$ cadence (N. Gehrels \& D. Palmer 2008, private communication); it reports the start time of the block containing the maximum, rather than making any 
attempt to identify the start of the burst, and does so with a $320 \mathrm{~ms}$ granularity. As another example, there have been reports of sub-threshold precursors to many GRBs (Burlon et al. 2009). For each GRB in our sample, we checked tens of seconds of light curve by eye to look for both excessive difference between the trigger time and the apparent rise time, and also for precursors, but found nothing to suggest that we should correct the published trigger times. The largest timing uncertainty we identified is the delay between the compact merger and the prompt emission of the internal shocks. We search for gravitational-wave signals within an on-source segment of $[-5,+1)$ s around each trigger time for each GRB of interest, feeling that this window captures the physical model with some tolerance for its uncertainties.

Because we believe that a gravitational wave associated with a GRB only occurs in the on-source segment, we use 324 off-source trials, each $6 \mathrm{~s}$ long, to estimate the distribution of background due to the accidental coincidences of noise triggers. We also re-analyze the off-source trials with simulated signals added to the data to test the response of our search to signals; these we call injection trials. The actual number of off-source trials included in the analysis varied by GRB, as the trials that overlapped with data-quality vetoes were discarded (Abbott et al. 2009b). To prevent biasing our background estimation due to a potential loud signal in the on-source trial, the off-source segments do not use data within $48 \mathrm{~s}$ of the on-source segment, reflecting the longest duration of templates in our bank. Finally, we discard $72 \mathrm{~s}$ of data subject to filter transients on both ends of the off-source region. Taking all of these requirements into account, the minimum analyzable time is $2190 \mathrm{~s}$.

\subsection{Sample Selection}

X-ray and $\gamma$-ray instruments identified a total of 212 GRBs during the S5 run, 211 with measured durations; 30 of them have a $T_{90}$ duration smaller than 2 s. $T_{90}$ is the time interval over which $90 \%$ of all counts from a GRB are recorded. While the $T_{90}$ classifies a burst as long or short, it is not a definitive discriminator of progenitor systems. In addition to the short GRBs, GRB 051211 (Kawai et al. 2005) and GRB 070714B (Barbier et al. 2007) are formally long GRBs, but they have spectral features hinting at an underlying coalescence progenitor. GRB 061210 is another long-duration burst, but it exhibits the typical short spikes of a short GRB (Cannizzo et al. 2006). This gives a list of 33 interesting GRBs with which to search for an association with gravitational waves from compact binary coalescence.

Around the trigger time of each interesting GRB, we required $2190 \mathrm{~s}$ of multiply coincident data. The detectors operated with individual duty cycles of $67 \%-81 \%$ over the span of the S5 and VSR1 runs. Where more than two detectors had sufficient data, we selected the most sensitive pair based on the average inspiral range, because including a third, less sensitive detector does not enhance the sensitivity greatly. The one exception was GRB 070923, described below. In descending order of sensitivity, the detectors are $\mathrm{H} 1, \mathrm{~L} 1, \mathrm{H} 2$, and V1. This procedure yielded 11 GRBs searched for in H1-L1 coincident data, 9 GRBs in $\mathrm{H} 1-\mathrm{H} 2$, and 1 in $\mathrm{H} 2-\mathrm{L} 1$.

In addition to these 21, we analyze GRB 070923 because of its sky location relative to the detectors' antenna patterns. The antenna pattern changes with the location of a source relative to a detector and can be expressed by the response $\sqrt{F_{+}^{2}+F_{\times}^{2}}$ in which $F_{+}$and $F_{\times}$denote the antenna-pattern functions (Allen et al. 2005). A value of 1 corresponds to an optimal location of the putative gravitational-wave source
Table 1

Parameters of the 22 GRBs Selected for this Search

\begin{tabular}{|c|c|c|c|}
\hline GRB & Redshift & Duration (s) & References \\
\hline 051114 & $\ldots$ & 2.2 & G4272, G4275 \\
\hline 051210 & $\ldots$ & 1.2 & G4315, G4321 \\
\hline 051211 & $\ldots$ & 4.8 & G4324, G4359 \\
\hline 060121 & $\ldots$ & 2.0 & G4550 \\
\hline 060313 & $<1.7$ & 0.7 & G4867, G4873, G4877 \\
\hline 060427B & $\ldots$ & 2.0 & G5030 \\
\hline 060429 & $\ldots$ & 0.25 & G5039 \\
\hline 061006 & $\ldots$ & 0.50 & G5699, G5704 \\
\hline 061201 & $\ldots$ & 0.80 & G5881, G5882 \\
\hline 061217 & 0.827 & 0.30 & G5926, G5930, G5965 \\
\hline 070201 & $\ldots$ & 0.15 & G6088, G6103 \\
\hline 070209 & $\ldots$ & 0.10 & G6086 \\
\hline 070429B & $\ldots$ & 0.50 & G6358, G6365 \\
\hline 070512 & $\ldots$ & 2.0 & G6408 \\
\hline 070707 & $\ldots$ & 1.1 & G6605, G6607 \\
\hline 070714 & $\ldots$ & 2.0 & G6622 \\
\hline 070714B & 0.92 & 64.0 & G6620, G6623, G6836 \\
\hline 070724 & 0.46 & 0.40 & G6654, G6656, G6665 \\
\hline 070729 & $\ldots$ & 0.90 & G6678, G6681 \\
\hline 070809 & $\ldots$ & 1.3 & G6728, G6732 \\
\hline 070810B & $\ldots$ & 0.08 & G6742, G6753 \\
\hline 070923 & $\ldots$ & 0.05 & G6818, G6821 \\
\hline
\end{tabular}

Notes. The values in the References column give the number of the GRB Coordinates Network (GCN) notice from which we took the preceding information (Barthelmy 2009).

relative to the observatory, while a value of 0 corresponds to a source location that will not induce any strain in the detector. For this particular GRB, the optimal antenna response for Virgo is around 0.7, while those for the two LIGO sites are about half of that (see Table 2), yielding a comparable sensitivity in the direction of GRB 070923 for all three of them. Data from H1, L1, and V1 were analyzed, making this the only GRB involving triple coincidences.

Table 1 lists all 22 target GRBs after applying the selection criteria described in this section. Plausible redshifts have been published for only three of these GRBs, placing them well outside of our detectors' range, but short GRB redshift determinations are in general sufficiently tentative to warrant searching for all of these GRBs.

GRB 070201 is also worth special mention. It was already analyzed in a high-priority search because of the striking spatial coincidence of this GRB with M31, a galaxy only $\sim 780 \mathrm{kpc}$ from Earth. No gravitational-wave signal was found and a coalescence scenario could be ruled out with $>99 \%$ confidence at that distance (Abbott et al. 2008a), lending additional support for a soft gamma repeater hypothesis (Ofek et al. 2008). However, because of improvements in the analysis pipeline, we re-analyzed this GRB and report the results in this paper. See Section 3.1.1 for details.

\subsection{Candidate Generation}

We generated candidates using the standard, untriggered compact binary coalescence search pipeline described in detail in Abbott et al. (2009b). The core of the inspiral search involves correlating the measured data against the theoretical waveforms expected from compact binary coalescence, a technique called matched filtering (Helmstrom 1968). The gravitational waves from the inspiral phase, when the binary orbit decays under gravitational-wave emission prior to merger, are accurately modeled by post-Newtonian approximants in the band of the de- 
tector's sensitivity for a wide range of binary masses (Blanchet 2006). The expected gravitational-wave signal, as measured by LIGO and Virgo, depends on the masses ( $\left.m_{\mathrm{NS}}, m_{\mathrm{comp}}\right)$ and spins $\left(\vec{s}_{\mathrm{NS}}, \vec{s}_{\text {comp }}\right)$ of the NS and its companion (either NS or BH), as well as the spatial location $(\alpha, \delta)$, inclination angle $\iota$, and polarization angle $\psi$ of the orbital axis. In general, the power of matched filtering depends most sensitively on accurately tracking the phase evolution of the signal. The phasing of compact binary inspiral signals depends on the masses and spins, the time of merger, and an overall phase.

We adopted a discrete bank of template waveforms that span a two-dimensional parameter space (one for each component mass) such that the maximum loss in signal-to-noise ratio $(\mathrm{S} / \mathrm{N}$ ) for a binary with negligible spins would be 3\% (Cokelaer 2007). While the spin is ignored in the template waveforms, we verify that the search can still detect binaries with most physically reasonable spin orientations and magnitudes with only moderate loss in sensitivity. For simplicity, the template bank is symmetric in component masses, spanning the range $[2,40) M_{\odot}$ in total mass. The number of template waveforms required to achieve this coverage depends on the detector noise spectrum; for the data analyzed in this paper the number of templates was around 7000 for each detector.

We filtered the data from each of the detectors through each template in the bank. If the matched filter $\mathrm{S} / \mathrm{N}$ exceeds a threshold, the template masses and the time of the maximum $\mathrm{S} / \mathrm{N}$ are recorded. For a given template, threshold crossings are clustered in time using a sliding window equal to the duration of the template (Allen et al. 2005). For each trigger identified in this way, the coalescence phase and the effective distance-the distance at which an optimally oriented and optimally located binary, with masses corresponding to those of the template, would give the observed $\mathrm{S} / \mathrm{N}$ - are also computed. Triggers identified in each detector are further required to be coincident with their time and mass parameters with a trigger from at least one other detector, taking into account the correlations between those parameters (Robinson et al. 2008). This significantly reduces the number of background triggers that arise from matched filtering in each detector independently.

The $\mathrm{S} / \mathrm{N}$ threshold for the matched filtering step was chosen differently depending on which detectors' data are available for a given GRB. If data from $\mathrm{H} 1$ and L1 were analyzed, the threshold for each detector was set to 4.25 , reflecting their comparable sensitivity. If data from $\mathrm{H} 1$ and $\mathrm{H} 2$ were analyzed, the threshold of the latter detector - the less sensitive of the two-was set to 3.5 to gain maximum network sensitivity, while the threshold of the more sensitive detector, $\mathrm{H} 1$, was set to 5.5 since any signal seen in $\mathrm{H} 2$ would be twice as loud in $\mathrm{H} 1$, with some uncertainty. In the single case of analyzing only H2-L1 data (GRB 070707) the threshold was 4.25 for L1 and 3.5 for $\mathrm{H} 2$, and for the single case of analyzing data with Virgo (GRB 070923), the threshold was set to 4.25 for all involved detectors (H1, L1, and V1). For comparison, a uniform S/N threshold of 5.5 was used in the untriggered S5 search (J. Abadie et al. 2010, in preparation). We applied two signal-based tests to reduce and refine our trigger sets. First, we computed a $\chi^{2}$ statistic (Allen 2005) to measure how different a trigger's $\mathrm{S} / \mathrm{N}$ integrand looks from that of a real signal; triggers with large $\chi^{2}$ were discarded. Second, we applied the $r^{2}$ veto (Rodríguez 2007) which discards triggers depending on the duration that the $\chi^{2}$ statistic stays above a threshold. The $\mathrm{S} / \mathrm{N}$ and $\chi^{2}$ from a single detector were combined into an effective $\mathrm{S} / \mathrm{N}$ (Abbott et al. 2008b). The effective S/Ns from the analyzed detectors were
Table 2

Summary of the Results for the Search for Gravitational Waves From Each GRB

\begin{tabular}{|c|c|c|c|c|c|c|c|}
\hline \multirow[t]{2}{*}{ GRB } & \multicolumn{4}{|c|}{ Antenna Response } & \multirow[b]{2}{*}{ F.A.P. } & \multicolumn{2}{|c|}{$\begin{array}{c}\text { Excluded Distance } \\
(\mathrm{Mpc})\end{array}$} \\
\hline & H1 & $\mathrm{H} 2$ & L1 & V1 & & NS-NS & $\mathrm{NS}-\mathrm{BH}$ \\
\hline 051114 & 0.56 & 0.56 & $\ldots$ & $\ldots$ & 1 & 2.3 & 6.2 \\
\hline 051210 & 0.61 & 0.61 & $\ldots$ & $\ldots$ & 0.10 & 3.3 & 4.3 \\
\hline 051211 & 0.53 & $\ldots$ & 0.62 & $\ldots$ & 0.66 & 2.3 & 8.9 \\
\hline 060121 & 0.11 & $\ldots$ & 0.09 & $\ldots$ & 0.58 & 0.4 & 1.3 \\
\hline 060313 & 0.59 & 0.59 & $\ldots$ & $\ldots$ & 0.16 & 1.4 & 4.3 \\
\hline 060427B & 0.91 & $\ldots$ & 0.92 & $\ldots$ & 1 & 7.0 & 12.7 \\
\hline 060429 & 0.92 & 0.92 & $\ldots$ & $\ldots$ & 0.21 & 4.3 & 6.2 \\
\hline 061006 & 0.61 & 0.61 & $\ldots$ & $\ldots$ & 1 & 2.3 & 8.2 \\
\hline 061201 & 0.85 & 0.85 & $\ldots$ & $\ldots$ & 1 & 4.3 & 10.1 \\
\hline 061217 & 0.77 & $\ldots$ & 0.52 & $\ldots$ & 0.23 & 3.2 & 11.8 \\
\hline 070201 & 0.43 & 0.43 & $\ldots$ & $\ldots$ & 0.07 & 3.3 & 5.3 \\
\hline 070209 & 0.19 & $\ldots$ & 0.12 & $\ldots$ & 0.76 & 2.3 & 4.2 \\
\hline 070429B & 0.99 & $\ldots$ & 0.93 & $\ldots$ & 0.31 & 8.9 & 14.6 \\
\hline 070512 & 0.38 & $\ldots$ & 0.51 & $\ldots$ & 0.97 & 6.1 & 8.9 \\
\hline 070707 & $\ldots$ & 0.87 & 0.79 & $\ldots$ & 0.87 & 4.2 & 7.1 \\
\hline 070714 & 0.28 & $\ldots$ & 0.40 & $\ldots$ & 0.72 & 4.2 & 2.3 \\
\hline 070714B & 0.25 & $\ldots$ & 0.38 & $\ldots$ & 0.54 & 3.2 & 5.1 \\
\hline 070724 & 0.53 & $\ldots$ & 0.70 & $\ldots$ & 0.84 & 5.1 & 11.8 \\
\hline 070729 & 0.85 & 0.85 & $\ldots$ & $\ldots$ & 0.40 & 7.0 & 10.8 \\
\hline 070809 & 0.30 & 0.30 & $\ldots$ & $\ldots$ & 1 & 2.3 & 4.3 \\
\hline 070810B & 0.55 & $\ldots$ & 0.34 & $\ldots$ & 0.50 & 2.3 & 6.1 \\
\hline 070923 & 0.32 & $\ldots$ & 0.40 & 0.69 & 0.74 & 5.1 & 7.9 \\
\hline
\end{tabular}

Notes. The antenna response column contains the response for each detector as explained in Section 2.2; an ellipsis (...) denotes that a detector's data were not used. F.A.P. is the false-alarm probability of the most significant on-source candidate for a GRB as measured against its off-source trials, as explained in Section 2.4. On-source trials with no candidates above threshold are assigned a F.A.P. of 1 . The last two columns show the lower limits at $90 \%$ CL on distances, explained in Section 3.2.

then added in quadrature to form a single quantity $\rho_{\text {eff }}^{2}$ which provided better separation between signal candidate events and background than $\mathrm{S} / \mathrm{N}$ alone. The list of coincident triggers at this stage are then called candidate events.

\subsection{Ranking Candidates}

The distribution of effective $\mathrm{S} / \mathrm{Ns}$ from background and from signals can vary strongly across the template bank, depending most strongly on the chirp mass, a combination of the two component masses that appears in the leading term of the signal amplitude and phase (Thorne 1987). For this reason, we refine our candidate ranking with a likelihood-ratio statistic, which we compute for every candidate in the on-source, off-source, and injection trials. In short, we define the likelihood ratio $L$ for a candidate $c$ to be the efficiency divided by the false-alarm probability. The efficiency here is the probability of obtaining a candidate as loud or louder than $c$ (by effective $\mathrm{S} / \mathrm{N}$ ) within the same region of template space given a signal in the data. The efficiency is a function of the signal parameters $m_{\text {comp }}$ and $D$ and is marginalized over all other parameters; it is obtained by simply counting across injection trials. The falsealarm probability here is the probability of obtaining a candidate as loud or louder than $c$ in the same region of chirp mass from noise alone; it is obtained by counting across off-source trials.

At the end of the search (i.e., Table 2 and Figure 1), we report a different false-alarm probability. It is the fraction of off-source likelihood ratios larger than the largest on-source likelihood ratio. 


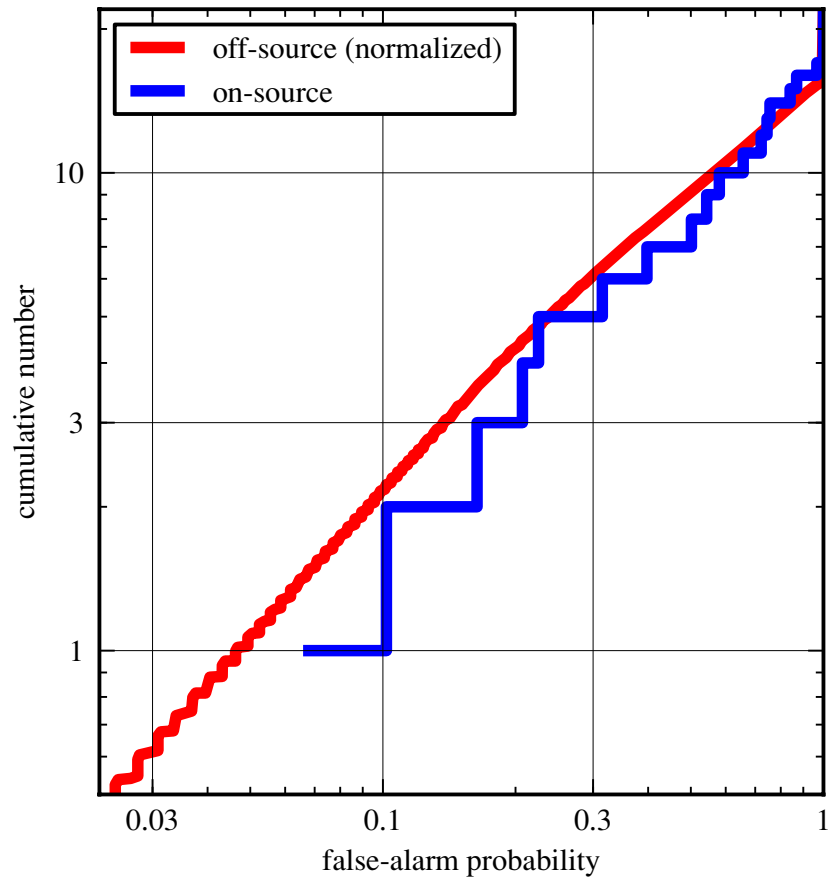

Figure 1. Cumulative false-alarm probabilities for the most significant candidate in each on- and off-source trial, as described in Section 2.4.

(A color version of this figure is available in the online journal.)

There is another noteworthy difference with respect to untriggered inspiral searches. For background estimation, untriggered searches use coincidences found between triggers from different detectors, to which unphysical time shifts greater than the light-travel time between detector sites have been applied. Unfortunately, $\mathrm{H} 1$ and $\mathrm{H} 2$, being co-located, share a common environmental noise that is absent from the time-shift background measurement. Being unable to estimate the significance of $\mathrm{H} 1-\mathrm{H} 2$ candidates reliably, the untriggered search examines them with significantly greater reservation and does not consider them at all in upper limit statements on rates. The present search performs its background estimation with unshifted coincidences under the assumption that any gravitational-wave signal will appear only in the on-source trial. Thus, we regain the unconditional use of $\mathrm{H} 1-\mathrm{H} 2$ candidates.

\section{RESULTS}

\subsection{Individual GRB Results}

We found no evidence for a gravitational-wave signal in coincidence with any GRB in our sample. We ran the search as described in the previous section and found that the loudest observed candidates in each GRB's on-source segment are consistent with the expectation from its off-source trials. The results are summarized in Table 2, with brief highlights in the following subsections. A graphical comparison of on-source to off-source false-alarm probability is shown in Figure 1.

\subsubsection{GRB 070201}

The re-analysis of GRB 070201 yielded candidates in the onsource segment, despite having no coincident candidate at all in the previous analysis (Abbott et al. 2008a). This is consistent because the threshold for $\mathrm{H} 2$ has been lowered from 4.0 to 3.5 and the coincident trigger found in this re-analysis happened to lie very close to the larger threshold in the previous search. The re-analysis yields a false-alarm probability of $6.8 \%$, the smallest in the set of analyzed GRBs. ${ }^{98}$ This value is completely within our expectations when we consider that we examined 22 GRBs.

\subsubsection{GRB 070923}

GRB 070923 was the GRB for which H1, L1, and V1 had comparable sensitivity and we accepted triggers from all three detectors. There were no triply coincident candidates in the on-source trial, but there were surviving doubly coincident candidates, the loudest of which had a false-alarm probability of $74.5 \%$.

\subsection{Distance Exclusions}

With our null observations and a large number of simulations, we can constrain the distance to each GRB assuming it was caused by a compact binary coalescence with a NS (with a mass in the range $[1,3) M_{\odot}$ ) and a companion of mass $m_{\text {comp }}$. For a given $m_{\text {comp }}$ range, we used the approach of Feldman \& Cousins (1998) to compute regions in distance where gravitationalwave events would, with a given confidence, have produced results inconsistent with our observations. Figure 2 shows the lower Feldman-Cousins distances for the 22 analyzed GRBs at $90 \%$ confidence for two illustrative choices for the companion mass range. The values are also listed in Table 2. Because the companion mass range has been divided into equally spaced bins, we report on an "NS-NS" system in which the companion mass is in the range $[1,4) M_{\odot}$ and an "NS-BH" system in which the $\mathrm{BH}$ has a mass in the range $[7,10) M_{\odot}$. The median exclusion distance for an NS-BH system is $6.7 \mathrm{Mpc}$, and for an NS-NS system it is $3.3 \mathrm{Mpc}$. These distances were derived assuming no beaming (uniform prior on $\cos \iota$ ). NS-BH distances are typically higher than NS-NS because more massive systems radiate more total gravitational-wave energy. The excluded distance depends on various parameters: the location of the GRB on the sky, the detectors used for the GRB, the noise floor of the data itself, and the likelihood ratio of the loudest on-source candidate event for the GRB.

We drew the simulations from a distribution in which our marginalized parameters roughly reflect our priors on these astrophysical compact binary systems. In our models, a signal is completely specified by $\left(m_{\mathrm{NS}}, m_{\text {comp }}, \vec{s}_{\mathrm{NS}}, \vec{s}_{\text {comp }}, \iota, \psi, t_{0}, D\right.$, $\alpha, \delta$ ). Of these, we wish to constrain $m_{\text {comp }}$ and $D$, marginalizing over everything else. We drew the NS mass $m_{\mathrm{NS}}$ uniformly from $[1,3) M_{\odot}$; the magnitudes of the NS spins $\left|\vec{s}_{\mathrm{NS}}\right|$ were half 0 and half uniform in $[0,0.75$ ) (Cook et al. 1994); the magnitudes of the companion's spin $\left|\vec{s}_{\text {comp }}\right|$ were half 0 and half uniform in $[0,0.98$ ) (Mandel \& O'Shaughnessy 2010); the orientations of the spins were uniform in solid angle; the inclination $\iota$ of the normal to the binary's orbital plane relative to our line of sight was conservatively chosen to be uniform in $\cos \iota$ instead of making an assumption about the GRB beaming angle; the polarization angle $\phi$ was uniform in $[0,2 \pi)$; the coalescence time $t_{0}$ was uniform over the off-source region; the declination $\delta$ was set to that of the GRB; the right ascension $\alpha$ was also set to that of the GRB, but was adjusted based on $t_{0}$ to keep each simulation at the same location relative to the detector as the GRB.

A number of systematic uncertainties enter into this analysis, but amplitude calibration error and Monte Carlo counting

\footnotetext{
98 In public presentations of preliminary results, GRB 061006 was erroneously highlighted as having the loudest candidate due to a $22.8 \mathrm{~s}$ offset in the GRB time. Swift's initial GCN alert (Schady et al. 2006a) was later corrected (Schady et al. 2006b), but we initially overlooked this correction.
} 


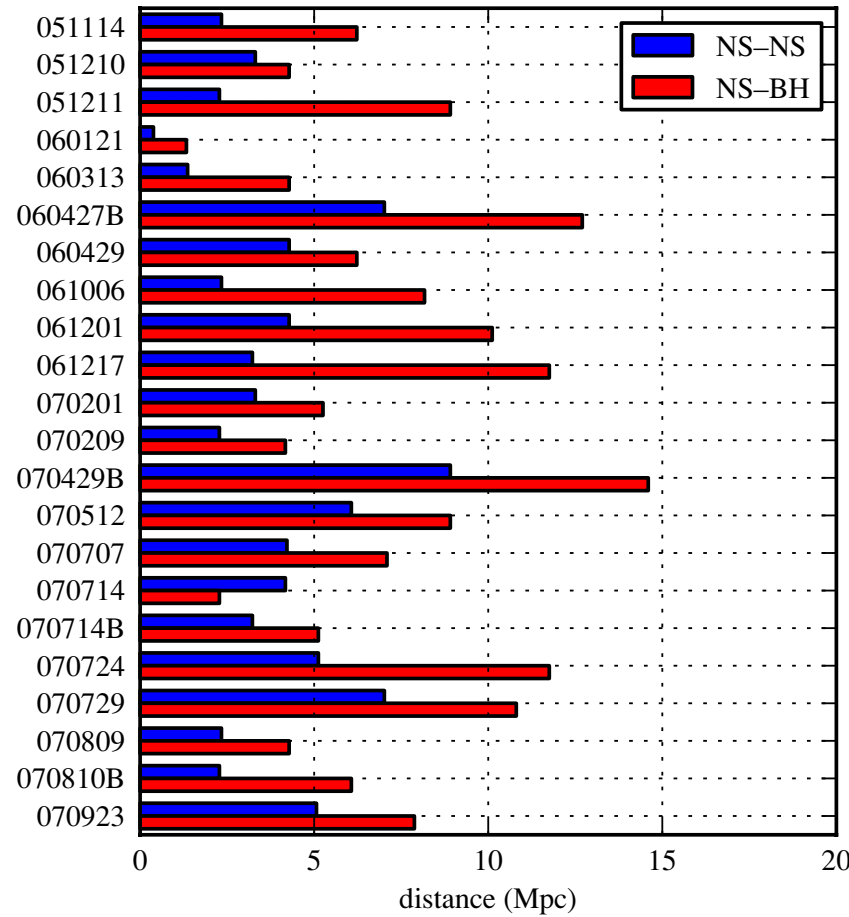

Figure 2. Lower limits on distances at $90 \%$ CL to putative NS-NS and NS-BH progenitor systems, as listed in Table 2 and explained in Section 3.2.

(A color version of this figure is available in the online journal.)

statistics from the injection trials have the largest effects. We multiplied exclusion distances by $1.28 \times\left(1+\delta_{\text {cal }}\right)$, where $\delta_{\text {cal }}$ is the fractional uncertainty $(10 \%$ for $\mathrm{H} 1$ and $\mathrm{H} 2 ; 13 \%$ for $\mathrm{L} 1 ; 6 \%$ for V1; Marion et al. 2008). The factor of 1.28 corresponds to a $90 \%$ pessimistic fluctuation, assuming Gaussianity. To take the counting statistics into account, we stretched the Feldman-Cousins confidence belts to cover the probability $\mathrm{CL}+1.28 \sqrt{\mathrm{CL}(1-\mathrm{CL}) / n}$, where CL is the desired confidence limit and $n$ is the number of simulations contained in the $\left(m_{\text {comp }}, D\right)$ bin for which we are constructing the belt.

\subsection{Population Statement}

In addition to the individual detection searches above, we would like to assess the presence of gravitational-wave signals that are too weak to stand out above background separately, but that are significant when the entire population of analyzed GRBs is taken together. We compare the cumulative distribution of the false-alarm probabilities of the on-source sample with the off-source sample. The on-source sample consist of the results of all 22 individual searches, including those for GRBs with known redshifts, and the off-source sample consists of 6801 results from the off-source trials. This number is lower than $22 \times 324$ because for some GRBs, some off-source trials were discarded due to known data-quality issues.

These two distributions are compared in Figure 1. To determine if they are consistent with being drawn from the same parent distribution, we employ the non-parametric Wilcoxon-Mann-Whitney $U$-statistic (Mann \& Whitney 1947), which is a measure of how different two populations are. Applying the $U$-test, we find that the two distributions are consistent with each other; if the on-source and off-source significances were drawn from the same distribution, they would yield a $U$-statistic greater than what we observed $53 \%$ of the time. Therefore, we find no evidence for an excess of weak gravitational-wave signals associated with GRBs.

\section{DISCUSSION}

We searched data taken with the three LIGO detectors and the Virgo detector for gravitational-wave signatures of compact binary coalescences associated with 22 GRBs but found none. We were sensitive to systems with total masses $2 M_{\odot}<m<$ $40 M_{\odot}$. We also searched for a population of signals too weak to be individually detected, but again found no evidence. While there are few redshift determinations for short GRBs, it appears that the distribution is peaked around $\langle z\rangle \sim 0.25$ (Nakar 2007), far outside initial detector sensitivity, so it is not surprising that the S5/VSR1 run yielded no detections associated with short GRBs.

We are indebted to the observers of the electromagnetic events and the GCN for providing us with valuable data. The authors gratefully acknowledge the support of the United States National Science Foundation for the construction and operation of the LIGO Laboratory, the Science and Technology Facilities Council of the United Kingdom, the Max-Planck-Society, and the State of Niedersachsen/Germany for support of the construction and operation of the GEO600 detector, and the Italian Istituto Nazionale di Fisica Nucleare and the French Centre National de la Recherche Scientifique for the construction and operation of the Virgo detector. The authors also gratefully acknowledge research support by these agencies and by the Australian Research Council, the Council of Scientific and Industrial Research of India, the Istituto Nazionale di Fisica Nucleare of Italy, the Spanish Ministerio de Educación y Ciencia, the Conselleria d'Economia Hisenda i Innovació of the Govern de les Illes Balears, the Foundation for Fundamental Research on Matter supported by the Netherlands Organisation for Scientific Research, the Royal Society, the Scottish Funding Council, the Scottish Universities Physics Alliance, The National Aeronautics and Space Administration, the Carnegie Trust, the Leverhulme Trust, the David and Lucile Packard Foundation, the Research Corporation, and the Alfred P. Sloan Foundation.

This document bears the LIGO document number P0900074.

\section{REFERENCES}

Abbott, B., et al. 2008a, ApJ, 681, 1419

Abbott, B., et al. 2008b, Phys. Rev. D, 77, 062002

Abbott, B., et al. 2009a, Rep. Prog. Phys., 72, 076901

Abbott, B., et al. 2009b, Phys. Rev. D, 79, 122001

Abbott, B., et al. 2010, ApJ, 715, 1438

Acernese, F., et al. 2008, Class. Quantum Grav., 25, 114045

Allen, B. 2005, Phys. Rev. D, 71, 062001

Allen, B., Anderson, W. G., Brady, P. R., Brown, D. A., \& Creighton, J. D. E. 2005, arXiv:gr-qc/0509116

Aptekar, R. L., et al. 1995, Space Sci. Rev., 71, 265

Atwood, W. B., et al. 2009, ApJ, 697, 1071

Barbier, L., et al. 2007, GCN Circ., 6623, 1

Barthelmy, S. D. 2009, The GCN Web site, http://gcn.gsfc.nasa.gov/gcn/

Blanchet, L. 2006, Living Rev. Rel., 9, 3 (http://www.livingreviews.org/lrr2006-4)

Bloom, J. S., Butler, N. R., \& Perley, D. A. 2008, in AIP Conf. Ser. 1000, Gamma Ray Bursts, ed. M. Galassi, D. Palmer, \& E. Fenimore (Melville, NY: AIP), 11

Burlon, D., Ghirlanda, G., Ghisellini, G., Greiner, J., \& Celotti, A. 2009, A\&A, 505,569

Campana, S., et al. 2006, Nature, 442, 1008

Cannizzo, J. K., et al. 2006, GCN Circ., 5904, 1

Chapman, R., Priddey, R. S., \& Tanvir, N. R. 2009, MNRAS, 395, 1515

Cokelaer, T. 2007, Phys. Rev. D, 76, 102004

Cook, G. B., Shapiro, S. L., \& Teukolsky, S. A. 1994, ApJ, 424, 823

Cutler, C., \& Flanagan, E. 1994, Phys. Rev. D, 49, 2658

Davies, M. B., Levan, A. J., \& King, A. R. 2005, MNRAS, 356, 54

Etienne, Z. B., et al. 2008, Phys. Rev. D, 77, 084002 
Faber, J. A., Baumgarte, T. W., Shapiro, S. L., Taniguchi, K., \& Rasio, F. A. 2006, AIP Conf. Proc., 861, 622

Feldman, G. J., \& Cousins, R. D. 1998, Phys. Rev. D, 57, 3873

Finn, L. S., \& Chernoff, D. F. 1993, Phys. Rev. D, 47, 2198

Flanagan, Éanna É., \& Hinderer, T. 2008, Phys. Rev. D, 77, 021502

Galama, T. J., et al. 1998, Nature, 395, 670

Gehrels, N., et al. 2004, ApJ, 611, 1005

Gehrels, N., et al. 2006, Nature, 444, 1044

Greiner, J. 2009, GRBs localized with BSAX or BATSE/RXTE or ASM/RXTE or IPN or HETE or INTEGRAL or Swift or AGILE or Fermi/GLAST, http://www.mpe.mpg.de/ jcg/grbgen.html

Helmstrom, C. W. 1968, Statistical Theory of Signal Detection (2nd ed.; London: Pergamon)

Hjorth, J., et al. 2003, Nature, 423, 847

Hurley, K., et al. 2005, Nature, 434, 1098

Kawai, N., et al. 2005, GCN Circ., 4359, 1

Klebesadel, R. W., Strong, I. B., \& Olson, R. A. 1973, ApJ, 182, L85

Kouveliotou, C., Meegan, C. A., Fishman, G. J., Bhat, N. P., Briggs, M. S., Koshut, T. M., Paciesas, W. S., \& Pendleton, G. N. 1993, ApJ, 413, L101

Malesani, D., et al. 2004, ApJ, 609, L5

Mandel, I., \& O'Shaughnessy, R. 2010, Class. Quantum Grav., in press (arXiv:0912.1074)

Mann, H. B., \& Whitney, D. R. 1947, Ann. Math. Statist., 18, 50

Marion, F., Mours, B., \& Rolland, L. 2008, h(t) Reconstruction for VSR1, Versions 2 and 3., Tech. Rep. VIR-078A-08, Virgo Project (https://tds.ego-gw.it/ $\mathrm{q} 1 / \mathrm{c}=2092$ )
Mészáros, P. 2006, Rep. Prog. Phys., 69, 2259

Nakar, E. 2007, Phys. Rep., 442, 166

Nakar, E., Gal-Yam, A., \& Fox, D. B. 2006, ApJ, 650, 281

Nissanke, S., Hughes, S. A., Holz, D. E., Dalal, N., \& Sievers, J. L. 2010, ApJ, submitted (arXiv:0904.1017)

Ofek, E. O., et al. 2008, ApJ, 681, 1464

Palmer, D. M., et al. 2005, Nature, 434, 1107

Poisson, E., \& Will, C. M. 1995, Phys. Rev. D, 52, 848

Read, J. S., Markakis, C., Shibata, M., Uryū, K., Creighton, J. D. E., \& Friedman, J. L. 2009, Phys. Rev. D, 79, 124033

Ricker, G. R., et al. 2003, in AIP Conf. Ser. 662, Gamma-Ray Burst and Afterglow Astronomy 2001: A Workshop Celebrating the First Year of the HETE Mission, ed. G. R. Ricker \& R. K. Vanderspek (Melville, NY: AIP), 3

Robinson, C. A. K., Sathyaprakash, B. S., \& Sengupta, A. S. 2008, Phys. Rev. D, 78, 062002

Rodríguez, A. 2007, Master's thesis, Louisiana State Univ. (http://arxiv.org/abs/ astro-ph/0612572)

Rosswog, S. 2007, RevMexAA Conf. Ser., 27, 57

Schady, P., et al. 2006a, GCN Circ., 5699,

Schady, P., et al. 2006b, GCN Rep., 6, 1

Shibata, M., \& Taniguchi, K. 2008, Phys. Rev. D, 77, 084015

Thorne, K. S. 1987, in Three Hundred Years of Gravitation, ed. S. W. Hawking \& W. Israel (Cambridge: Cambridge Univ. Press), 330

Will, C. M. 2005, Living Rev. Rel., 9, 3 (http://www.livingreviews.org/lrr-2006-3)

Winkler, C., et al. 2003, A\&A, 411, L1

Zhang, B., Zhang, B. B., Liang, E. W., Gehrels, N., Burrows, D. N., \& Mészáros, P. 2007, ApJ, 655, L25 